\title{
The Effect of a Discrete Signal on Context Conditioning: Assessment by Preference and Freezing Tests
}

\author{
J. H. RoAld Maes \\ University of Nijmegen, Nijmegen, The Netherlands
}

AND

VINCENT M. LOLORDO

Dalhousie University, Halifax, Nova Scotia, Canada

\begin{abstract}
Four experiments with rats assessed conditioning to contextual cues after the delivery of footshocks that were either signaled by a discrete stimulus or unsignaled. Two different tests were used. The first was a context preference test in which subjects were allowed to move freely in a brightly lit, unconditionally aversive context and the former shock context. The second test consisted of scoring freezing behavior while the animals were confined to the former conditioning context. During context preference tests, signaled-shock animals spent more time in the conditioning context and/or entered that context more frequently than did unsignaled-shock subjects. However, freezing tests largely failed to detect a difference between groups. These results were discussed in terms of possible interactions between the formation of contextshock, signal-shock, and context-signal associations and their effect on performance in each of the two types of tests. () 1996 Academic Press, Inc.
\end{abstract}

A number of formal models of associative learning predict that the presence of a discrete conditioned stimulus (CS) during classical conditioning has a detrimental effect on the formation of an association between static background stimuli, or context, and the unconditioned stimulus (US) (e.g., Mackintosh, 1975; Pearce \& Hall, 1980; Rescorla \& Wagner, 1972). That is, the CS-

This research was supported by a grant from the Netherlands Organization for Scientific Research to J. H. R. Maes for a 1-year postdoctoral visit to Dalhousie University, and a grant from the Natural Sciences and Engineering Research Council of Canada to V. M. LoLordo. We thank R. C. Barnet for his valuable comments on an earlier version of this article. Correspondence should be addressed to J. H. R. Maes, Department of Comparative and Physiological Psychology, University of Nijmegen, P.O. Box 9104, 6500 HE Nijmegen, The Netherlands. E-mail: maes@ nici.kun.nl. 
US association "overshadows" the context-US association and the context's excitatory strength is less than if the US had not been signaled by the CS.

Research testing this prediction has produced mixed results. Odling-Smee (1975a,b, 1978a,b) reported a series of studies in which rats received a shock while they were confined to the small, black compartment of a two-compartment box. The rats had previously demonstrated a preference for this black compartment over the larger, white compartment. The shock in the black box was signaled by a CS for some animals and unsignaled for others. After conditioning, the excitatory strength of the black box was assessed by a context preference test in which the animals were allowed to move freely in both compartments. Odling-Smee found that rats that had a predictive CS present during conditioning spent more time in the black compartment than did animals that had received unsignaled shocks. This result was interpreted as reflecting the potential of a CS to interfere with the formation of an association between the black box and shock.

Recently, failures to find a difference in contextual excitation between signaled-US and unsignaled-US conditions have been reported by Balsam and Gibbon (1988), Williams and LoLordo (1995), and Williams, Frame, and LoLordo (1992). Balsam and Gibbon employed an autoshaping procedure with pigeons, whereas Williams et al. used rats as subjects, a shock as a US, and freezing, a species-specific defence reaction, as a measure of context conditioning (see also, e.g., Sigmundi \& Bolles, 1983).

The conflicting results of Odling-Smee's versus the Williams et al. experiments are especially noteworthy. In both types of research, rats were used as subjects, and a footshock served as the US. Perhaps the only essential difference is the nature of the dependent measure employed (see also Williams, Frame, \& LoLordo, 1991). If so, then the question arises as to which one of the two measures reflects the "true" associative strength of the context after signaled and unsignaled shocks.

One possibility is that freezing is a less sensitive measure than context preference for detecting differences in associative strength. In other words, perhaps the context preference test provides the better assessment. Although this possibility cannot be ruled out on the basis of previous research, this line of reasoning seems to be at odds with the outcome of studies in which freezing has been directly compared with other conventional indices of conditioning, such as suppression of bar pressing for food. At least with discrete auditory CSs, high correlations among freezing and these other measures have typically been found (e.g., Bouton \& Bolles, 1980; Mast, Blanchard, \& Blanchard, 1982, Sigmundi, Bouton, \& Bolles, 1980; see also Fanselow, Kim, Yipp, \& De Oca, 1994, and Young \& Fanselow, 1992, for additional support for the notion of freezing as a sensitive measure).

For several reasons, one could argue instead that it is the context preference measure that does not reflect the context's true associative strength. First, Balsam and Gibbon (1988) pointed to the possibility that the results of the 
context preference tests in Odling-Smee's studies may show only that rats prefer signaled events over unsignaled events, regardless of the nature of the US (either appetitive or aversive; see, e.g., Dinsmoor, 1983). On this view, it may be a mistake to interpret the outcomes of the context preference tests in terms of differences in the strength of a context-US association. Second, it may be that the unconditioned preference for dark shown in a light/dark twocompartment apparatus is increased by fear (Aitken, 1974; Allison, Larson, \& Jensen, 1967). Thus, a long time spent in the former shock context would not necessarily reflect limited context conditioning. Third, perhaps freezing that occurs while an animal is in the black box during a context preference test results in the animal spending a relatively long time in the former shock context. If this happens, then the inference that the former shock context's excitatory strength is low would be incorrect (see also Fanselow, DeCola, \& Young, 1993). With regard to Odling-Smee's finding that signaled-shock rats spent more time in the black box than unsignaled-shock rats, the latter two arguments actually imply that the signaled-shock rats were more fearful than the unsignaled-shock rats.

The primary goal of the present experiments was to examine the relationship between rats' performance in context preference tests on the one hand, and freezing in the conditioning context on the other, after signaled versus unsignaled exposures to a shock US. Specifically, we aimed at empirically establishing within experiments that one can reliably detect a difference between signaled-shock and unsignaled-shock animals using a context preference measure, but not on the basis of a freezing measure. This was achieved by conducting both types of test separately within each experiment. Also, freezing in the shock context was continuously monitored while the animals were tested for their preference for the contexts. By doing so, the contribution of freezing to the performance on each context preference test could be evaluated. On the basis of the results of the present experiments, a tentative evaluation of the viability of the accounts outlined above and, consequently, of an overshadowing interpretation of the results of context preference tests is offered under General Discussion.

\section{EXPERIMENT 1}

In the first experiment, rats received a context preference test in a twocompartment box that was modeled after that used by Odling-Smee. After it was established that the rats preferred to stay in the black box, one-half of the rats received tone-shock trials in the black compartment. The other half received unsignaled shocks. All animals were then tested for fear of the black box by placing them in the white compartment and allowing them to move freely throughout both compartments. The dependent measures of this test were the number of times that the animals entered the black box and the total time that they spent there. Finally, the rats were again confined to the black box and freezing behavior was scored, while no CSs or USs were presented. 
If the two tests are equally sensitive, and performance during both the context preference test and the freezing test only reflects the effect of a context-shock association, then the tests should reveal similar results. If, however, the two measures tap different mechanisms or associations and/or they are not equally sensitive to detecting differences in the strength of contextual excitation, it is conceivable that, as in previous research by Odling-Smee, the context preference test will reveal a greater preference for the black box in signaled-shock animals than in unsignaled-shock animals, whereas the freezing test, as in the Williams et al. experiments, will reveal no difference between these two types of subjects.

\section{METHOD}

\section{Subjects}

The subjects were 20 male Sprague-Dawley rats obtained from Charles River, Canada. The animals were approximately 4 months old at the start of the experiment. Four of the animals were experimentally naive and 16 rats had previously participated in a study in which they had received 2 unsignaled electric shocks $(0.8 \mathrm{~mA}, 1 \mathrm{~s})$, and a total of $0-18$ nonreinforced 30 -s tone $(82 \mathrm{~dB}, 7 \mathrm{kHz})$ presentations. Both these shocks and tones had been presented in contexts distinctively different from the white and black boxes used in the present study. Each of the 16 animals used previously had received extinction sessions in its former conditioning context and had shown no freezing in that context at the end of these sessions.

The animals were individually housed in wire mesh cages with ad lib food and water. They were maintained on a 16-h/8-h light/dark cycle. The experimental manipulations were conducted during the light portion of the cycle.

\section{Apparatus}

A two-compartment box like that used by Odling-Smee (1975a,b, 1978a,b) was employed in the present research. One compartment measured $40 \times 40$ $\times 20 \mathrm{~cm}$. The walls and the floor of this compartment were made of plywood and painted glossy white. It was covered by a clear Plexiglas lid. The smaller compartment $(20 \times 13 \times 18 \mathrm{~cm})$ consisted of mat-black plywood walls, a clear Plexiglas lid, and a grid floor. The grid was made of 12 2-mm stainless steel rods. These rods were connected to a Grason-Stadler shock generator that provided a $0.4-\mathrm{mA}, 1-\mathrm{s}$ scrambled-shock US. The two compartments could be separated by a clear Plexiglas sliding door. A speaker hung from the ceiling of the experimental room at a distance of $2 \mathrm{~m}$ above the black compartment. This speaker was used for the presentation of a 82-dB (C), 3$\mathrm{kHz}$ tone that served as a CS for one-half of the subjects. A 65-dB (C) background noise was provided by a ventilation fan. Illumination was provided by two $100-\mathrm{W}$ overhead light bulbs. 
During all phases of the experiment, a Panasonic video camera was placed above the black box to record the rat's behavior inside. An auditory stimulus was superimposed on the videotapes. This stimulus was presented once every $5 \mathrm{~s}$ and was used for pacing the scoring of the rat's behavior (see Procedure).

\section{Procedure}

The first three stages of the procedure were similar to those in OdlingSmee's experiments.

In Stage 1 of the experiment, the subjects were individually placed in the white compartment. The animals were allowed to freely explore both of the compartments for $5 \mathrm{~min}$. A rat was considered to be "in" the black box if it had all four paws in the box. As soon as the animal subsequently had at least two paws in the white compartment, it was considered to have left the black box. The dependent measures of this test and of the other context preference tests performed during Stage 3 (see below) were the total number of times an animal entered the black compartment and the total time spent there. The reliability of the scoring on the frequency measure of the context preference tests was checked by a second observer who was unaware of the purpose of the experiments. For each rat, this observer scored the number of entries into the black box during the initial context preference test of Experiment 2. The reliability expressed in terms of Pearson's correlation coefficient ( $r$ ) was $r=0.97$. During the first context preference test, the subjects spent at least $175 \mathrm{~s}$ in the black box. Two matched groups were formed $(n=10)$ on the basis of the two dependent measures and prior experimental history.

Stage 2, which started on the following day, consisted of the conditioning session. Rats were individually confined to the black compartment for 25 min. Group U (unsignaled shock) received 10 shock USs presented at a variable interval (mean, 137 s). Group $S$ (signaled shock) received exactly the same treatment as did Group U, except that each US was preceded by a tone CS. The CS was $10 \mathrm{~s}$ in duration and the shock was presented during the last second of the tone presentation.

Immediately after the conditioning session, the door separating the two compartments was raised and for $5 \mathrm{~min}$ the animal could move freely throughout the two compartments. This phase (a "stabilization period") was used by Odling-Smee to diminish freezing behavior during the context preference tests of Stage 3.

The first context preference test of Stage 3, called context preference Test 2, was performed approximately $2 \mathrm{~min}$ after the stabilization period. The test was identical to that performed in Stage 1. Approximately $24 \mathrm{~h}$ thereafter, an identical context preference test, Test 3, was conducted. The apparatus had been cleaned with tap water prior to each of the context preference tests.

The rat's behavior while it was in the black box during each of the latter two context preference tests was scored as "freezing" or "not freezing" once every $5 \mathrm{~s}$. Freezing was defined as the absence of visible movement of 
the body and the vibrissae. The only movement that was tolerated was movement related to respiration. All other behavior was scored as not freezing. The reliability of the scoring of freezing presented in this article was checked by the same second observer mentioned above. Of each experiment, this observer rescored freezing of at least two freezing or context preference tests (total of nine test sessions). For each of these sessions, a Pearson's correlation coefficient was computed using the data from the two observers. The mean correlation coefficient was $r=.94$ (SEM, 0.2).

Stage 4 commenced $24 \mathrm{~h}$ after the last context preference test. Each rat was confined to the black box for $5 \mathrm{~min}$. During this period, the rat's behavior was scored as freezing or not freezing. No CSs or USs were scheduled to occur in this stage. The black box was cleaned with tap water between sessions.

\section{Data Analyses}

Each of the experiments reported below was performed in two replications. A Replication factor was initially included in all analyses of variance (ANOVAs). Unless stated otherwise, this factor did not significantly interact with any other factor of primary interest and was, therefore, excluded from the analyses.

The results of the context preference tests in Experiment 1 were analyzed by Wilcoxon rank-sum tests with a correction for ties. A nonparametric test was employed because of the large incidence of zero scores. The data of all other tests in this experiment, and the data of Experiments 2-4, were analyzed parametrically by means of ANOVAs. In each of the experiments, the relationship between freezing and context preference scores was examined by computing Pearson correlation coefficients between the percentage of observations that were scored as freezing while the animal was in the black box during a context preference test on the one hand, and the total number of entries into the black box, or the total time spent there, on the other. A significance level of $p<.05$ was used in all statistical analyses.

\section{RESULTS}

The left side of Fig. 1 shows the median time that the groups spent in the black compartment during each of the three context preference tests. The figure shows that after conditioning, both groups spent considerably less time in this compartment than they did in the initial preference test. However, during the third context preference test, but not during the second test, Group $\mathrm{S}$ appeared to spend more time in the conditioning context than did Group $\mathrm{U}$. The right side of Fig. 1 depicts the median number of times each group went into the black box during the context preference tests. Group S entered the conditioning box more often than did Group $U$ on the third context preference test.

Analyses revealed that during the third test, Group S entered the black 

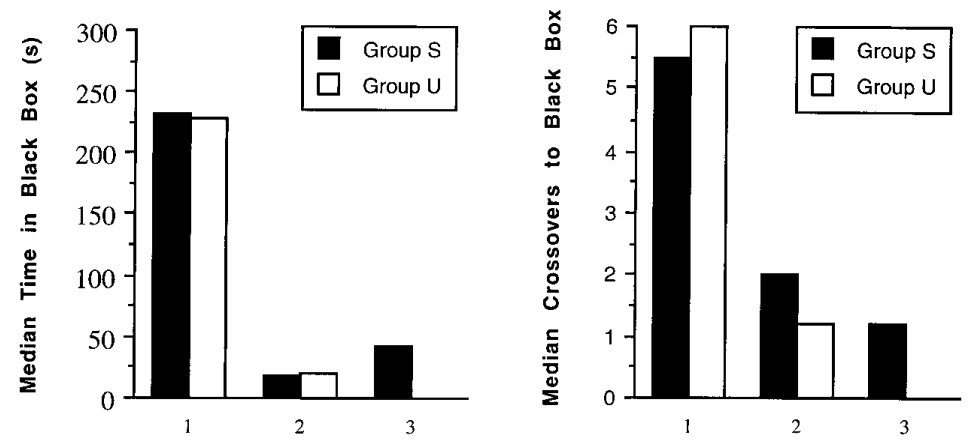

Context Preference Test

FIG. 1. (Left) Groups' median time spent in the black box during the context preference tests of Experiment 1. (Right) Groups' median number of entries into the black box during the preference tests of Experiment 1. Tests 2 and 3 were performed, respectively, $7 \mathrm{~min}$ and $24 \mathrm{~h}$ after the conditioning session in the black box.

compartment more often and spent more time there than did Group U (Wilcoxon rank-sum tests, $z \mathrm{~s}>1.99)$. On the second context preference test, there was no difference between groups on either of the two measures $(z \mathrm{~s}<.19)$.

An examination of the relationship between freezing in the black box during the third context preference test on the one hand and the total time spent there on the other revealed the following pattern. In Group U, only 2 of 10 animals entered the black box. Each of these animals subsequently stayed in this context for most of the 5-min test (202 and $265 \mathrm{~s})$ and showed high levels of freezing. Thus, one animal froze on $83.3 \%$ of the observations made in the black box; the other animal froze on 58.5\%. In Group S, there were only 3 animals that did not enter the black box during the third test. The remaining 7 animals in this group that did enter the black compartment stayed there for a mean time of $175.8 \mathrm{~s}$. The 3 animals of Group $\mathrm{S}$ that spent at least $267 \mathrm{~s}$ in the black box also had the highest freezing percentages (40.7, 71.9, and 75.4), whereas the remaining 4 animals of Group $S$ that spent less than $114 \mathrm{~s}$ in the black box, had a mean freezing percentage of only $1.9 \%$. Collectively, for the animals in the two groups that did enter the black box, there was a significant positive correlation between the total amount of time spent in the black box and the percentage of observations while they were there scored as freezing $(r(8)=.87)$. In contrast, the correlation between the number of entries and freezing in the shock context was not significant $(r(8)$ $=-.35$ ).

Despite the observed difference between groups on the third context preference test, no difference in freezing was observed on the next day when the 
animals were confined to the black box. Group S froze on $52.8 \%$ of the observations, and Group U on $60.2 \%$. A one-factor ANOVA failed to detect a difference between groups $(F(1,18)<1)$.

\section{DISCUSSION}

As in Odling-Smee's studies, signaled-shock rats spent more time in the black box than did unsignaled-shock animals. However, unlike Odling-Smee's findings, the difference between groups became apparent only on the test that was performed 1 day after the conditioning session and not during the preference test performed several minutes after conditioning. This difference may have to do with the relatively high shock intensity used in the present experiment. The current parameters were chosen to decrease the chance of a floor effect with respect to detecting differences between the two groups during the freezing test. However, the present shock intensity is somewhat less optimal for detecting a difference between groups on a context preference measure than that suggested by Odling-Smee's findings (1975b).

Given the intermediate level of freezing during the freezing test, the null result with respect to this test cannot be attributed to floor or ceiling effects. Furthermore, the null result is especially noteworthy in the sense that Group S did not even show attenuated freezing after having had a longer nonreinforced exposure to the black box than Group U. (Group S spent more time in the black box during the third context preference test than did Group U.)

The results of Experiment 1 illustrate the state of affairs with respect to the literature on overshadowing of context by a discrete CS. That is, context preference shows the effect under conditions where freezing does not.

The present experiment also provides a first indication as to the viability of the notion that the results of context preference tests are confounded by freezing in the black box (see introduction). Although it is true that for the rats that did enter the black box, the total time spent there was positively correlated with freezing, the differences between Group S and Group U on the two preference measures cannot be attributed solely to differences in freezing. First, the majority of the subjects of Group U completely avoided the black box. Second, the crossover score was not significantly correlated with freezing.

\section{EXPERIMENT 2}

Experiment 2 was intended to assess whether or not a difference in freezing between signaled-shock and unsignaled shock subjects will emerge when they are tested at the very same times that the critical context preference tests were performed in Experiment 1. It might be that first performing the context preference tests prior to the freezing test, as was done in the previous experiment, somehow reduced the sensitivity of the freezing test. Alternatively, the difference between groups in freezing might only emerge $24 \mathrm{~h}$ after the conditioning session, and not after $48 \mathrm{~h}$. 
Two additional tests were performed in Experiment 2. One test was a context-preference test and was conducted to determine whether or not a difference between groups would still emerge after the two nonreinforced freezing tests (extinction sessions). In the second test, the associative strength of the tone was assessed in a neutral test context. The purpose of this latter test was to provide an index of conditioning to the CS that could then be correlated with freezing scores and the context preference scores. By doing so, further insight into the relationship between the various measures could be obtained.

\section{METHOD}

\section{Subjects and Apparatus}

Twenty-four experimentally naive male Sprague-Dawley rats, obtained from the same supplier as the rats in Experiment 1, were used. Their mean weight was $377 \mathrm{~g}$ (range, 344-422 g). Housing and maintenance conditions were as in Experiment 1. The same two-compartment box, US, and CS were employed as in the previous experiment. To test the associative strength of the tone, a context was used that was distinctively different from the twocompartment apparatus. It consisted of a box (dimensions: $23 \times 16 \times 24$ $\mathrm{cm})$ that had aluminum back and side walls. The front wall and ceiling were made of clear Plexiglas. The floor was composed of 17 stainless-steel rods that were $2 \mathrm{~mm}$ in diameter, spaced $1.9 \mathrm{~cm}$ apart. There were two of these boxes and they were placed in a room that was illuminated by a $40-\mathrm{W}$ red overhead light. A distinctive odor cue was provided by cleaning the boxes with a solution of ammonia and water prior to each session. The same ventilation fan as used when the animals were in the two-compartment box provided a background noise. The tone was presented through the same speaker as was used for conditioning in the signaled-shock group. The speaker hung from the ceiling at a distance of approximately $1.5 \mathrm{~m}$ from the CS test box.

\section{Procedure}

Stage 1 and the conditioning and stabilization sessions of Stage 2 were the same as described for Experiment 1. Thus, the animals first received a 5-min context preference test. Subsequently, two groups were formed, matched on the basis of the number of crossings and the total time spent in the black box. During the following session, 10 shocks were delivered while the animals were confined to the black box. These shocks were either signaled (Group S) or unsignaled (Group U). This session was followed by a 5-min stabilization session during which the animal could move freely throughout the two compartments. Instead of performing a context preference test 2 min after the stabilization period, each animal was confined to the black box for $5 \mathrm{~min}$ and its behavior was scored as freezing or not freezing, as was done in Stage 4 of Experiment 1. Twenty-four hours later, a second freezing test was 


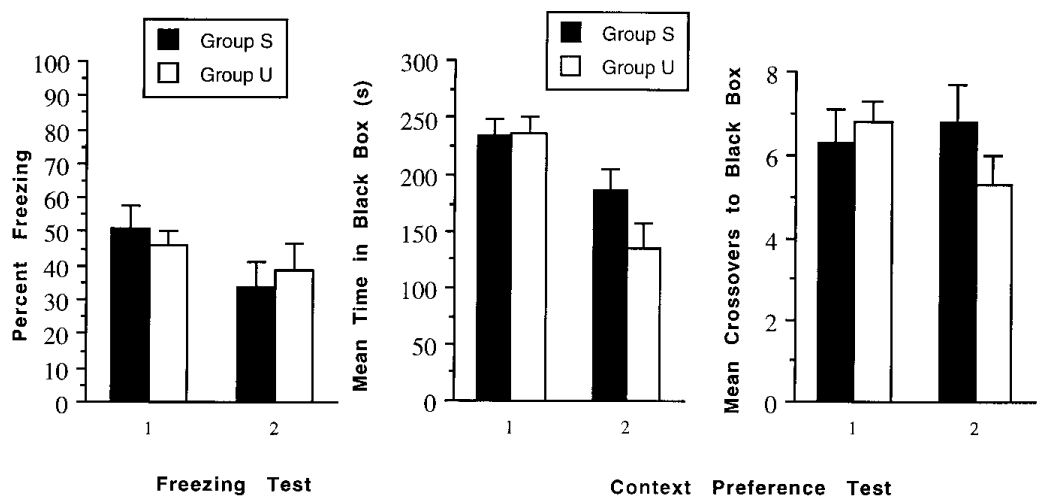

FIG. 2. (Left) Mean percentage $(+S E M)$ of samples scored as freezing during freezing Tests 1 and 2 of Experiment 2 . Test 1 was performed 7 min after conditioning and Test 2 was conducted $24 \mathrm{~h}$ later. (Middle) Mean time $(+S E M)$ spent in the black box during context preference Tests 1 and 2 of Experiment 2. Test 2 was performed after the last freezing test. (Right) Mean number of times $(+S E M)$ the groups entered the black box during context preference Tests 1 and 2 of Experiment 2.

performed that was identical to the first. On the next day, a context preference test was performed as in Stage 1. Finally, animals were individually placed in the CS test context, $4 \mathrm{~h}$ after the last context preference test. Four tones were presented during this test at a mean interval of $245 \mathrm{~s}$. The duration of each tone presentation was increased to $30 \mathrm{~s}$ in order to acquire more data points per rat using the same 5-s time sampling technique to assess freezing as was used in the previous stages. Freezing during the 30 -s period prior to each CS presentation was assessed (pre-CS freezing), as was freezing during each 30-s CS presentation (CS freezing).

\section{RESULTS}

The left side of Fig. 2 shows the percentage of samples that were scored as freezing during the freezing tests. As can be seen, there was no clear difference between groups on either test. A Group $\times$ Replication $\times$ Test repeated measures ANOVA revealed a significant effect of Test $(F(1,20)=$ 4.94), which reflects the diminished freezing during Test 2, relative to Test 1. Furthermore, the Group $\times$ Replication interaction was also significant $(F(1,20)=11.39)$. Simple main effect analyses revealed that the interaction was caused by Group $U$ showing a higher level of freezing across the two test sessions than Group $S$ in the first replication $(F(1,20)=5.70)$ and Group $\mathrm{S}$ showing more freezing than Group $\mathrm{U}$ in the second replication $(F(1,20)=$ 5.70). No other main effects or interactions were significant. The reason for the difference between replications is not clear. However, it is clear that the freezing measure did not reveal a consistent difference between groups. 
The middle panel of Fig. 2 depicts the mean time that each group spent in the black box during the initial context preference test and during the context preference test performed after the last freezing test. During the second preference test, Group $S$ appeared to spend more time in the shock context than did Group U. A Group $\times$ Test repeated measures ANOVA revealed a significant interaction $(F(1,22)=5.01)$. Subsequent simple main effect analyses using the error terms from the overall analysis (Winer, 1971) revealed that Group $\mathrm{S}$ spent more time in the black box than did Group U during Test $2(F(1,35)=4.58)$, but not during Test $1(F<1)$.

The number of crossovers during each of the two context preference tests are shown on the right side of Fig. 2. A Group $\times$ Test ANOVA did not detect any significant main or interaction effects $(F \mathrm{~s}(1,22)<2.37)$.

For each rat, the percentage of observations that were defined as freezing while the animal was in the black box during the second context preference test was determined. The mean percentage was 7.0 for Group $U$ and 9.6 for Group S. There was no significant difference between these two percentages $(F<1)$. There was a significant positive correlation between freezing in the black box during the second, critical context preference test and time spent in this box for Group $\mathrm{S}(r(11)=.78)$, but not for Group $\mathrm{U}(r(11)=.50)$. Likewise, the negative correlation between freezing during the context preference test and the number of crossovers during this test was significant for Group $\mathrm{S}(r(11)=-.83)$, but not for Group $\mathrm{U}(r(11)=-.44)$.

During the test for responding to the tone in the novel context, Group $S$ clearly showed a conditioned response to the tone. Pre-CS freezing in each of the groups was low (mean of $4.2 \%$ in Group S and of $2.8 \%$ in Group U). However, Group $\mathrm{S}$ froze much more during the tone presentations (mean, $51.3 \%$ ), whereas Group U did not (mean, $7.3 \%$ ). A Group $\times$ Period (pre-CS, CS) ANOVA revealed a significant effect of Group and Period and of the interaction between Group and Period $(F \mathrm{~s}(1,22)>25.42)$.

For the animals of Group S, the correlation between CS freezing on the one hand and freezing during the second freezing test, freezing during the second context preference test, the total time spent in the black box during preference Test 2, and the number of crossovers during preference Test 2 on the other were $.56, p=.06 ; .42, p>.1 ; .40, p>.1$; and $-.70, p<.01$, respectively.

\section{DISCUSSION}

The difference that emerged in Experiment 1 between Group S and Group $\mathrm{U}$ during the context preference test performed $24 \mathrm{~h}$ after conditioning was not paralleled in the present experiment by a difference between groups on the score of the freezing test that was also performed $24 \mathrm{~h}$ after conditioning. Hence, the null result of the freezing test in the previous experiment was not caused by the prior occurrence of the context preference tests or by the fact 
that the freezing test in that experiment occurred $48 \mathrm{~h}$ after conditioning instead of after $24 \mathrm{~h}$.

The context preference test revealed a difference between groups, even though the animals had been subjected to two freezing tests in which extinction of fear could occur. This finding illustrates the sensitivity of this measure for detecting a difference between signaled-shock and unsignaled-shock rats. Furthermore, as in Experiment 1, differences in context preference performance were not an artifact of differences in freezing during the preference test. The overall levels of freezing during the preference test were fairly low and did not differ between groups. Furthermore, although there were significant correlations between freezing and context preference measures, they were found only for one group, Group S. This means that freezing during the context preference test was not a factor that generally contributed to or determined the scores on the preference measures.

In Group S, CS freezing was positively correlated with context freezing during the last freezing test and negatively correlated with the number of crossovers during the last preference test. A possible interpretation of these correlations will be offered under General Discussion.

\section{EXPERIMENT 3}

The aim of Experiments 3 and 4 was to compare further the ability of context preference measures on the one hand and the freezing measure on the other, to reveal a difference in contextual excitation between rats receiving signaled versus unsignaled shocks. Only two shock trials were presented in each of the following two experiments. This is the minimum number of trials that, according to most learning models, is necessary to obtain overshadowing.

The conditioning parameters employed in Experiments 3 and 4 were identical to those used in recent experiments (Maes, Fidler, \& LoLordo, 1996) that failed to consistently reveal less contextual freezing after signaled shocks than after unsignaled shocks. It is of interest to determine whether or not a difference can be detected under these conditions when using a context preference test. In Experiment 3, two context preference tests were followed by a freezing test, whereas in Experiment 4, two freezing tests were followed by a context preference test.

\section{Method}

Twenty-four experimentally naive male Sprague-Dawley rats (mean weight, $330 \mathrm{~g}$; range, 281-428 g) obtained from Charles River, Canada, served as the subjects. Housing and maintenance conditions were as described in Experiment 1. The apparatus used was the same black/white two-compartment box as used in the previous experiments. The CS used was a 30-s, 7$\mathrm{kHz}$ tone. The procedure was exactly the same as that employed in Experiment 1, except for the following. In Stage 2, only two shocks were presented in a 5-min conditioning session. The first shock occurred $2.5 \mathrm{~min}$ after placement 

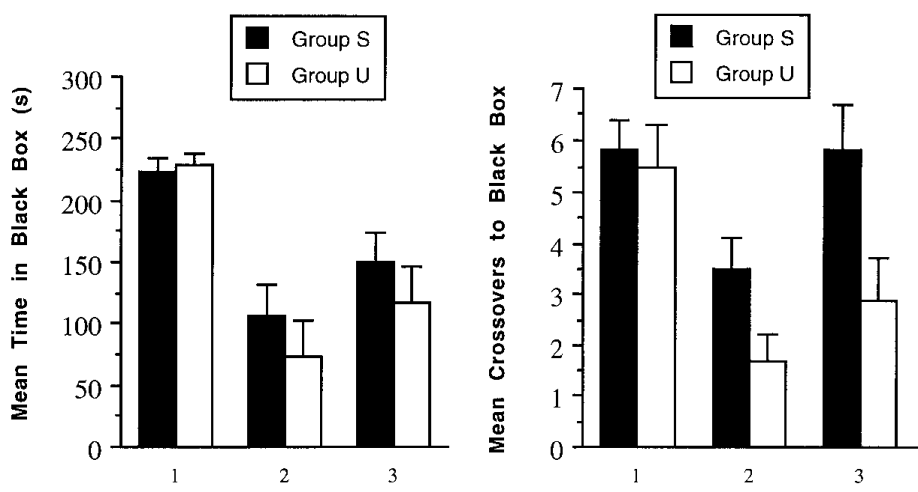

Context Preference Test

FIG. 3. (Left) Groups' mean time spent $(+S E M)$ in the black shock compartment during each of the context preference tests of Experiment 3. (Right) Groups' mean number ( $+S E M)$ of entries into the black box during the preference tests performed in Experiment 3. Tests 2 and 3 were performed $7 \mathrm{~min}$ and $24 \mathrm{~h}$ after conditioning, respectively.

into the black box, and the second shock was delivered 1.5 min after the first. The shocks were unsignaled in Group $\mathrm{U}(n=12)$. In Group $\mathrm{S}(n=12)$, each of the shocks was signaled by the 30 -s tone. The shock occurred during the last second of each tone presentation.

\section{RESULTS}

The left side of Fig. 3 depicts the mean time that each two groups spent in the black box during the initial context preference test and during the two preference tests performed after the conditioning stage. The right side of the figure shows the mean number of times that the groups entered the black, shock compartment during each of these three context preference tests. A Group $\times$ Test ANOVA performed on the duration data of the two context preference tests performed after conditioning, Tests 2 and 3, detected a significant effect only of Test $(F(1,22)=6.47$, other $F \mathrm{~s}<1)$. This reflects the increase in the total time spent in the black box from Test 2 to Test 3. An identical ANOVA performed on the crossover data from the same tests revealed that both groups entered the black box more often on Test 3 than on Test $2(F(1,22)=12.20)$. More importantly, across tests, Group S entered the black box more often than Group $U(F(1,22)=7.34)$.

The percentages of observations scored as freezing while the animals were in the black box during the preference tests were low. During context preference Test 2, 8 animals of Group $U$ entered the black box at least once and these animals froze on $4.1 \%$ of the observations made in this box. In Group $\mathrm{S}$, the corresponding percentage was $3.6 \%$ and was based on 11 subjects. 
The difference between the groups was not significant $(F(1,17)<1)$. During preference Test 3, 9 animals of Group $U$ and 11 animals of Group S entered the black box at least once. The percentages of observations scored as freezing in the black box were $17.4 \%$ for Group $U$ and $7.8 \%$ for Group S. Again, the difference was not significant $(F(1,18)=2.69)$.

There was a significant positive correlation between freezing in the black box during the third context preference test and time spent in this box for Group $\mathrm{S}(r(10)=.65)$ and a significant negative correlation between these two measures for Group $\mathrm{U}(r(8)=-.71)$. The negative correlation between freezing during the third context preference test and the number of crossovers during this test was significant for Group $\mathrm{U}(r(8)=-.68)$, but not for Group $\mathrm{S}(r(10)=-.07)$.

The percentage of observations scored as freezing while the subjects were confined to the black box during the freezing test was $31.9 \%$ for Group U and $18.9 \%$ for Group S. An ANOVA failed to detect a difference between the groups $(F(1,22)=1.83)$.

\section{DISCUSSION}

Experiment 3 basically replicates and extends the outcomes of Experiment 1. Even with the use of only two conditioning trials, a difference between signaled-shock and unsignaled-shock animals was observed on the crossover measure, but not on the freezing measure. Furthermore, the observed difference in performance during the preference tests again was not simply a result of a difference in freezing during these tests. Freezing levels were low and did not differ between groups and the score on the preference measure that in this experiment was most sensitive for detecting a between-groups difference, namely the crossover measure, was significantly correlated only with contextpreference test freezing for Group U.

\section{EXPERIMENT 4}

Experiment 4 assessed freezing to the conditioning context at precisely the same times in the sequence of events that the context preference tests had been performed in Experiment 3. Thus, Test 1 was performed 2 min after the stabilization phase, and Test 2 was conducted approximately $24 \mathrm{~h}$ after conditioning. The question of primary interest was whether these tests, as did the context preference tests in Experiment 3, would reveal a difference between signaled-shock and unsignaled-shock subjects. The experiment was concluded by performing a context preference test and a test for responding to the CS in an associatively neutral context.

\section{METHOD}

Twenty-four naive male Sprague-Dawley rats (mean weight: $358 \mathrm{~g}$ ) received a context preference test, a conditioning session, and a stabilization session as described in Experiment 3. The animals in Group $S(n=12)$ 


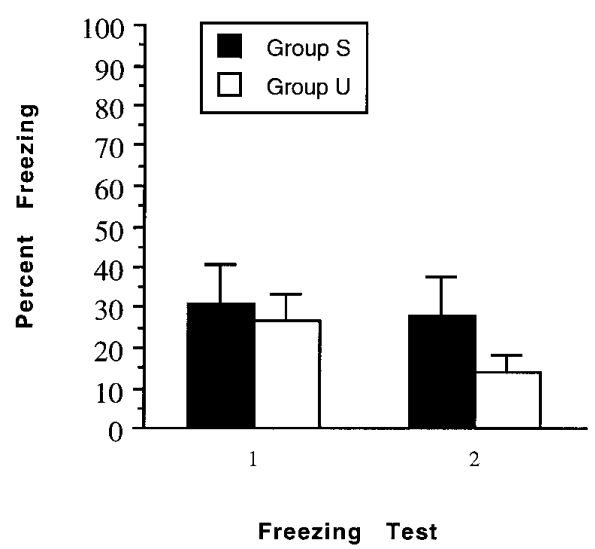

FIG. 4. Mean percentage $(+S E M)$ of observations scored as freezing during freezing Tests 1 and 2 of Experiment 4. Test 1 was performed $7 \mathrm{~min}$ after conditioning, and Test 2, $24 \mathrm{~h}$ after conditioning.

received tone-signaled shocks, whereas the animals in Group $\mathrm{U}(n=12)$ received unsignaled shocks. Two minutes after the stabilization phase, a 5min freezing test was performed. An additional freezing test was performed $24 \mathrm{~h}$ later. On the following day, a context preference test was conducted. Finally, approximately $4 \mathrm{~h}$ after this context preference test, a test for conditioned responding to the tone was performed in the same tone-test context as described in Experiment 2. Further experimental details with respect to conditioning, preference testing, and tests for freezing were as described for the previous experiment. The details of the CS test were as in Experiment 2.

\section{RESULTS}

The results of the freezing tests are shown in Fig. 4. A Group $\times$ Test repeated measures ANOVA using the freezing data failed to detect any significant main or interaction effects $(F \mathrm{~s}(1,22)<1.91)$.

During the first preference test, Group S spent $241.6 \mathrm{~s}$ in the black box; during the second context preference test, the total time spent there was 202.5 s. For Group U, the respective scores on the duration measure were 245.3 and 176.9 s. A Group $\times$ Test repeated measures ANOVA performed on the duration scores only detected a significant effect of Test $(F(1,22)=23.48)$. For Group S, the mean numbers of crossovers during the first and second context preference tests were 6.1 and 5.7, respectively. The means for Group $\mathrm{U}$ were 6.3 for Test 1 and 7.3 for Test 2. A Group $\times$ Test ANOVA on these crossover data did not reveal any reliable main or interaction effects $(F \mathrm{~s}(1,22)$ $<1.21)$.

During the second context preference test, the animals of Group S froze on $16.5 \%$ of the observations that were made while they were in the black 
box. For Group U, this percentage was $8.3 \%$. The difference was not reliable $(F(1,22)=1.21)$. There was a positive and significant correlation between second context preference test freezing and the total time spent in the black box for Group $\mathrm{S}(r(11)=.64)$ and a negative but nonsignificant correlation between these measures for Group $\mathrm{U}(r(11)=-.17)$. The correlation between freezing and the number of crossovers was negative and significant for both Group $\mathrm{S}(r(11)=-.77)$ and Group $\mathrm{U}(r(11)=-.64)$.

During the 30-s pre-CS periods of the test for responding to the tone, Group $\mathrm{U}$ froze on $5.6 \%$ of the measurements. During the CS periods the mean percentage for this group was $3.1 \%$. For Group S, the corresponding percentages were 4.5 and $18.8 \%$. A Group $\times$ Period (pre-CS/CS) repeated measures ANOVA only revealed a significant interaction between Group and Period $(F(1,22)=6.56)$, which reflects the fact that CS freezing was greater than pre-CS freezing in Group S, but not in Group U.

The correlations in Group S between CS freezing on the one hand and freezing during the last context freezing test, freezing during the last context preference test, total time spent in the black box during the last preference test, and the number of crossovers to the black box during preference Test 2 on the other were $.76, p<.01 ; .57, p=.06 ; .06, p>.8$; and $-.58, p<$ .05 , respectively.

\section{DISCUSSION}

In Experiment 3, a difference between groups was observed on the frequency score of the context preference tests that were conducted both several minutes after conditioning and $24 \mathrm{~h}$ thereafter. The null results of the freezing tests of the present experiment suggest that the failure to observe a difference in freezing in Experiment 3, which was assessed $48 \mathrm{~h}$ after conditioning, was not caused by conducting the test at an inappropriate time. In Experiment 4, the freezing tests were performed at exactly the same times after conditioning as the preference tests had been conducted in Experiment 3.

The context preference test in the present experiment was performed after two 5-min freezing tests. As the level of fear was relatively low immediately after conditioning to begin with, because only two shocks were used, the remaining level of fear after two subsequent extinction sessions was probably too low for a preference test to be able to detect group differences.

The pattern of correlations between CS freezing on the one hand and context freezing, total time spent in the black box during the second context preference test, and the number of crossovers to the black box during the second context preference test on the other matched those obtained in Experiment 2. A possible theoretical implication of this pattern will be discussed under General Discussion.

\section{GENERAL DISCUSSION}

In the present experiments, rats received shocks that were either signaled by a tone or unsignaled. Context preference tests were performed in which 
each rat could choose between entering the context in which it had received the shocks and staying in another context for which it had previously demonstrated an aversion. It was observed that signaled-shock animals spent more time in the shock compartment and/or they entered this compartment more often than did unsignaled-shock rats. Furthermore, context preference test performance was related to freezing that occurred in the shock context during testing, although this relationship was fairly complex and inconsistent between experiments. Differences in freezing could not completely account for the observed between-group differences on the two context preference measures. In none of the experiments was there a significant difference between signaled-shock and unsignaled-shock animals in the level of freezing shown during context preference tests or during confinement to the shock context.

One could argue that the rats' performance during the critical context preference tests was determined by freezing in the white, nonshock compartment. On this view, during the initial context preference test, the rats learned that the black and white compartments were part of one place. Consequently, fear that was subsequently conditioned to the black compartment generalized to the white compartment. When the animals were placed in the white compartment at the start of a context preference test that was performed after conditioning, the animals from the unsignaled-shock groups froze more in the non-shock compartment than did the subjects in the signaled-shock groups. This was because the subjects in the former group had acquired more context fear than the subjects in the latter group. As a result, the animals in the unsignaled-shock groups crossed over to the shock compartment less readily than the animals in the signaled-shock groups.

Although we did not measure freezing in the white compartment, this account can be rejected on the basis of the data present. The problem with this interpretation, which rests on the assumption of a strong generalization between compartments, is that the animals during the initial context preference test proved to discriminate very well between compartments. Furthermore, if freezing in the nonshock compartment is significantly and positively related to fear and freezing in the shock compartment, then, given the fact that we observed between-group differences on context preference tests, one would expect between-group differences in freezing in both nonshock and shock compartments. However, there were no between-group differences in freezing in the shock compartment before, during and after context preference tests. This absence of differences was not due to ceiling effects. In fact, in Experiments 2 and 3, low levels of freezing were found in the shock box to begin with, and, again, there were no differences between groups.

Similar problems arise when one tries to apply an account proposed by Aitken (1974) and Allison, Larson, and Jensen (1967) to the present data. On that account, the signaled-shock groups showed a higher preference for dark places than did the unsignaled-shock groups because they were more fearful. This account implies that the signaled-shock rat had formed a stronger 
shock box-US association than had the unsignaled-shock animals. This enhanced conditioned fear of the black compartment generalized to the white compartment. The generalized fear of the white compartment caused the signaled-shock subjects to demonstrate a larger preference for the black box, relative to the unsignaled-shock subjects, despite the fact that there existed a strong black box-US association in the signaled-shock rats. However, as already indicated above, the present data are problematic for the notion of generalization between compartments. Furthermore, this notion implies that neither freezing tests nor context preference tests yield a reliable index of context conditioning.

The dissociation between the effect of signaled versus unsignaled shocks on freezing on the one hand and performance during context preference tests on the other may be accounted for in at least two ways. First, performance of signaled-shock rats in the context preference tests might not have been determined by a reduced context-US association. Instead, perhaps the attractiveness of a signaled-shock context was increased, relative to an unsignaledshock context, because of the information provided by the signal (e.g., Balsam \& Gibbon, 1988; see also Berlyne, 1960). According to this account, which can readily explain the results of Experiments 1-4, a conflict was induced because the animals had to make a choice between staying in a large, brightly lit, and unconditionally aversive environment and entering a context in which an aversive event had previously occurred. Animals in the signaledshock group found the latter option more attractive than did the unsignaledshock animals. This was caused by the fact that signaled-shock animals had received more precise information about the occurrence of the shock than had unsignaled-shock rats. However, the association between context and shock was equally strong in the two groups, as was indicated by the equal freezing levels.

Although the notion that rats have a general preference for a context in which a biologically significant event is signaled over one in which it is unsignaled is able to account for the preference data, it is silent about the underlying mechanism of this preference. It remains unspecified how the availability of more precise information about the occurrence of the event in signaled-shock rats enhances the preference for the conditioning context, relative to the preference demonstrated by unsignaled-shock rats. It may be that signaled-shock animals use the additional information, for instance, to "prepare' themselves for the occurrence of the event and hence make the event less aversive (preparation hypothesis; Perkins, 1968) or to be able to "relax" between signal presentations (safety hypothesis; Mowrer, 1960). However, each of these two hypotheses actually implies that during the freezing tests, signaled-shock animals should demonstrate less fear than do unsignaled-shock animals. For further arguments against the information, preparatory-response, and safety-signal hypotheses, see Fanselow (1980).

A second account adopts the following assumptions. First, freezing is as 
sensitive a measure for assessing associative strength as is a context preference test or any other conventional test. Second, a CS-US association overshadows a context-US association. Third, if a discrete CS is present that signals the US, a context-CS association is also formed, at least under certain circumstances (see, e.g., Maes, Fidler, \& LoLordo, 1996, Marlin, 1982; Miller, McCoy, Kelly, \& Bardo, 1987; Rescorla, 1984). The existence of such an association in the present experiments is suggested by the positive correlation between freezing to the tone and freezing to the context, and the negative correlation between freezing to tone and the number of crossovers to the shock context that were found for the signaled-shock groups in Experiments 2 and 4, the two experiments in which there was a measure of freezing to tone in a neutral context. A context-CS association could compensate for the loss of a context-US association as a result of an overshadowing CSUS association. Context would evoke the CS representation, which in turn would evoke the US representation. This mechanism provides an alternative pathway to activation of the US representation. Fourth, and most importantly, in order for a context to evoke a representation of the CS, the temporal and contextual cues currently present have to match the cues that were present during conditioning to a substantial degree. Instead, for a context to evoke a representation of the US, temporal and contextual cues present may not have to resemble the cues present during conditioning as much as is the case for the evocation of a CS representation. In other words, the generalization gradient based on conditioned responding to a context that is associated with a US is flatter than is the case for a context associated with a CS.

There is some indirect support for this latter assumption from a study on human cardiac conditioning. Bersh, Notterman, and Schoenfeld (1956) reported the results of an experiment in which a decrease in heart rate was classically conditioned in human subjects. A tone was paired with a shock of either 20 or $28 \mathrm{~V}$. After conditioning, nonreinforced tones that differed in varying degrees from the tone used during conditioning in terms of frequency were presented. A generalization gradient could thus be determined. It was found that the generalization gradient was broader following conditioning with the stronger shock. A flattening of the generalization gradient of context freezing with increased shock intensity was also found in an experiment by Fanselow (1981; Fig. 4). These studies are relevant to the present discussion if it is assumed that the CS in our experiments is comparable to the weak US.

There is also more direct empirical support from our experiments that specifically relates to the above-mentioned fourth assumption. On two occasions, freezing to the shock context was attenuated for a limited time right after confinement of the animals of Group S to the shock context. In Experiment 1, Group $\mathrm{S}$ froze on $26.7 \%$ of the observations during the very first $30 \mathrm{~s}$ of the freezing test, and Group U on 55.0\%. An ANOVA performed on these initial freezing data revealed a marginally significant difference between groups 
$(F(1,18)=3.53, p=.056)$. An ANOVA performed on the freezing data of the first $30 \mathrm{~s}$ of the second freezing test of Experiment 2 (26.4\% freezing for Group $U$ and $8.3 \%$ freezing for Group S) revealed a significant difference between groups $(F(1,22)=4.57)$. These findings may indicate that the representation of the CS in Group $\mathrm{S}$ requires at least $30 \mathrm{~s}$ to become active to such an extent that the resultant excitatory strength becomes indistinguishable from the excitation in Group $\mathrm{U}$ (which, in turn, results from a context-US association that becomes active immediately after placement in the former conditioning context). During conditioning for Group S rats, the first toneshock pairing occurred $129 \mathrm{~s}$ after they were placed in the black compartment. Thus, the situation present just after placement in that compartment in the freezing test can be considered a generalized (temporal) CS. Our fourth assumption is that there would be less generalization based on the context-CS association in Group $S$ than there would be based on the context-US association in Group $\mathrm{U}$ and thus less fear in the former.

With the proposed set of assumptions it is possible to explain the present results. In Experiment 1, Group U had acquired a strong context-US association. Instead, Group S had acquired a relatively weak context-US association and, in addition, a context-CS association and a CS-shock association. During context preference testing after conditioning, most of the animals of Group $\mathrm{U}$ did not enter the black box at all. A typical behavior was that an animal started exploring the interior of the black compartment with its head partly in that compartment while its four paws remained in the white compartment. The notion put forward here is that the stimuli that the animal was confronted with in this manner were sufficiently similar to the stimuli that would have been present had the animals fully entered the black box, to evoke a representation of the US. Since rats tend to avoid spatially localized aversive stimuli, these rats tended to remain in the white box.

It must be noted that this account is not necessarily incompatible with our rejection of the idea that performance during the critical context preference tests was determined by freezing in the white, nonshock compartment. Avoidance of spatially localized stimuli does not necessarily rest on freezing in the place where the animal currently is (i.e., where it has its four paws). The form of the conditioned response, which in turn is a result of the activation of a US representation that is evoked by avoidable dangerous stimuli, may well be different from that evoked by unavoidable stimuli.

For the animals of Group S, the contextual stimuli present while partly exploring the interior of the black box with their paws still in the white box, evoked a representation of the CS, but, according to our fourth assumption, not to such an extent that they completely avoided entering the black compartment. However, after a short time in the black box, the CS representation became sufficiently active (although perhaps not yet completely active as they were now in the box with the door open instead of closed, as had been the case during conditioning) for the context to become aversive. Consequently, 
the animal left the black box. For some animals of Group S, this cycle repeated itself a few times; others stayed out of the black box after their first visit. During the test for freezing, all animals were confined to the black box and all respective representations were completely activated in each group. In Group S, the context-CS association, CS-US association, and possibly a weak context-US association supported a conditioned response that was indistinguishable from that supported by the strong context-US association in Group U.

This line of reasoning can also be applied to the results of Experiments 2 and 3, although here, the rats in Group $U$ entered the shock context at least once. This happened because the context-US association was weaker than in Experiment 1 as a result of the use of fewer conditioning trials, or due to partial extinction caused by performing freezing tests. However, in each of the experiments, the context-US association in Group $U$ became activated more easily than the context-CS association in Group S. Consequently, the subjects in Group $U$ were less prone than those in Group $S$ to either leave the shock compartment (the animals started freezing) or re-enter the compartment after having left it. This resulted in a net lower score on the duration measure and/or the crossover score in Group U than in Group S. However, for both groups the stimuli present during the freezing test exactly matched those present during conditioning, and the resultant activation of stimulus representations caused equal levels of freezing in the two groups.

The foregoing analysis implies that extinguishing a context-CS association and/or a CS-US association in the signaled-shock groups, should result in an even larger preference for the black box and less context freezing than is the case without such an extinction treatment. It must be noted that the proposed context-CS association, in principle, could have been extinguished in Experiments 2 and 4, as a result of first performing freezing (i.e., extinction) tests. However, the unsignaled-shock animals also received nonreinforced context exposures during the freezing tests of these experiments. This, in turn, means that the strength of the appropriate association, namely, a context-CS association for signaled-shock rats and a context-US association for unsignaled-shock rats, was attenuated for both groups of rats. So one might expect that the absolute difference between groups on the context preference measures would be largely the same, regardless of test order, which was the case.

As to the question of which of the two tests, a context preference test or a freezing test, provides the "better" assessment of contextual fear after signaled versus unsignaled shocks, the proposed account suggests that it depends on the specific association that one is interested in. The context preference test may provide a more "pure" measure of the strength of a contextUS association in that it is less confounded by the influence of a contextCS association than is the freezing measure. Alternatively, if the overall level of fear as a result of all underlying types of associations is of major concern, then the freezing test constitutes the preferred test. 


\section{REFERENCES}

Aitken, P. P. (1974). Aversive stimulation and rats' preference for areas differing in noveltyvalue and brightness. Animal Behavior, 22, 731-734.

Allison, J., Larson, D., \& Jensen, D. D. (1967). Acquired fear, brightness preference, and oneway shuttlebox performance. Psychonomic Science, 8, 269-270.

Balsam, P. D., \& Gibbon, J. (1988). Formation of tone-US associations does not interfere with the formation of context-US associations in pigeons. Journal of Experimental Psychology: Animal Behavior Processes, 14, 401-412.

Berlyne, D. E. (1960). Conflict, arousal, and curiosity. New York: McGraw-Hill.

Bersh, P. J., Notterman, J. M., \& Schoenfeld, W. N. (1956). Generalization to varying tone frequencies as a function of intensity of unconditioned stimulus. Texas: Air University, School of Aviation Medicine, USAF, Randolph AFB. [Cited in G. A. Kimble (1960), Hilgard and Marquis' conditioning and learning (2nd ed, p. 341). New York: Appleton].

Bouton, M., \& Bolles, R. C. (1980). Conditioned fear assessed by freezing and by the suppression of three different baselines. Animal Learning and Behavior, 8, 429-434.

Dinsmoor, J. A. (1983). Observing and conditioned reinforcement. Behavioral and Brain Sciences, 6, 693-728.

Fanselow, M. S. (1980). Signaled shock-free periods and preference for signaled shock. Journal of Experimental Psychology: Animal Behavior Processes, 6, 65-80.

Fanselow, M. S. (1981). Naloxone and Pavlovian fear conditioning. Learning and Motivation, 12, 398-419.

Fanselow, M. S., DeCola, J. P., \& Young, S. L. (1993). Mechanisms responsible for reduced contextual conditioning with massed unsignaled unconditioned stimuli. Journal of Experimental Psychology: Animal Behavior Processes, 19, 121-137.

Fanselow, M. S., Kim, J. J., Yipp, J., \& De Oca, B. (1994). Differential effects of n-methyl-daspartate antagonist dl-2-amino-5-phosphonovalerate on acquisition of fear of auditory and contextual cues. Behavioral Neuroscience, 108, 235-240.

Mackintosh, N. J. (1975). A theory of attention: Variations in the associability of stimuli with reinforcements. Psychological Review, 82, 276-298.

Maes, J. H. R., Fidler, T. L., \& LoLordo, V. M. (1996). Contextual fear after signalled versus unsignalled shocks: Effect of extent of prior experience with context and signal. Quarterly Journal of Experimental Psychology 49B, 174-187.

Marlin, N. A. (1982). Within-compound associations between the context and the conditioned stimulus. Learning and Motivation, 13, 526-541.

Mast, M., Blanchard, R. J., \& Blanchard, D. C. (1982). The relationship of freezing and response suppression in a CER situation. The Psychological Record, 32, 151-167.

Miller, J. S., McCoy, D. F., Kelly, K. S., \& Bardo, M. T. (1987). Within-compound associations between taste and contextual stimuli. Bulletin of the Psychonomic Society, 25, 124-125.

Mowrer, O. H. (1960). Learning theory and the symbolic process. New York: Wiley.

Odling-Smee, F. J. (1975a). Background stimuli and the interstimulus interval during Pavlovian conditioning. Quarterly Journal of Experimental Psychology, 27, 387-392.

Odling-Smee, F. J. (1975b). The role of background stimuli during Pavlovian conditioning. Quarterly Journal of Experimental Psychology, 27, 387-392.

Odling-Smee, F. J. (1978a). The overshadowing of background stimuli by an informative stimulus in aversive Pavlovian conditioning. Animal Learning and Behavior, 6, 43-51.

Odling-Smee, F. J. (1978b). The overshadowing of background stimuli: Some effects of varying amounts of training and UCS duration. Quarterly Journal of Experimental Psychology, 30, $737-746$.

Pearce, J. M., \& Hall, G. (1980). A model for Pavlovian learning: Variations in the effectiveness of conditioned but not of unconditioned stimuli. Psychological Review, 87, 532-552.

Perkins, C. C., Jr. (1968). An analysis of the concept of reinforcement. Psychological Review, 75, $155-172$. 
Rescorla, R. A. (1984). Associations between Pavlovian CSs and context. Journal of Experimental Psychology: Animal Behavior Processes, 10, 195-204.

Rescorla, R. A., \& Wagner, A. R. (1972). A theory of Pavlovian conditioning. Variations in the effectiveness of reinforcement and nonreinforcement. In A. H. Black \& W. F. Prokasy (Eds.), Classical conditioning. II. Current theory and research (pp. 105-132). New York: Appleton-Century-Crofts.

Sigmundi, R. A., \& Bolles, R. C. (1983). CS modality, context conditioning, and conditioned freezing. Animal Learning and Behavior, 11, 205-212.

Sigmundi, R. A., Bouton, M. E., \& Bolles, R. C. (1980). Conditioned freezing in the rat as a function of shock intensity and CS modality. Bulletin of the Psychonomic Society, 15, 254256.

Williams, D. A., \& LoLordo, V. M. (1995). Time cues block the CS, but the CS does not block time cues. Quarterly Journal of Experimental Psychology, 48B, 97-116.

Williams, D. A., Frame, K. A., \& LoLordo, V. M. (1991). Reexamination of contextual conditioning with massed versus distributed unconditioned stimuli. Journal of Experimental Psychology: Animal Behavior Processes, 17, 202-209.

Williams, D. A., Frame, K. A., \& LoLordo, V. M. (1992). Discrete signals for the unconditioned stimulus fail to overshadow contextual or temporal conditioning. Journal of Experimental Psychology: Animal Behavior Processes, 18, 41-55.

Winer, B. J. (1971). Statistical principles in experimental design (2nd ed.). New York: McGrawHill.

Young, S. L., \& Fanselow, M. (1992). Associative regulation of Pavlovian fear conditioning: Unconditional stimulus intensity, incentive shifts, and latent inhibition. Journal of Experimental Psychology: Animal Behavior Processes, 18, 400-413.

Received June 9, 1995

Revised February 2, 1996 